\title{
Classical and exotic magnetism: Recent advances and perspectives
}

Yuri Kivshar, and Andrew P. Roberts

Citation: Low Temperature Physics 43, 895 (2017); doi: 10.1063/1.5001281

View online: https://doi.org/10.1063/1.5001281

View Table of Contents: http://aip.scitation.org/toc/ltp/43/8

Published by the American Institute of Physics

\section{Articles you may be interested in}

Victor Valentinovich Eremenko (1932-2017) in honor of his 85th birthday

Low Temperature Physics 43, 893 (2017); 10.1063/1.5001280

Observation of new magnetic ground state in frustrated quantum antiferromagnet spin-liquid system $\mathrm{Cs}_{2} \mathrm{CuCl}_{4}$ Low Temperature Physics 43, 901 (2017); 10.1063/1.5001282

The magnon BEC observation by switch off method Low Temperature Physics 43, 930 (2017); 10.1063/1.5001292

Single crystals growth of hexaferrits $\mathrm{M}$-type $\mathrm{MTi}_{x} \mathrm{Co}_{x} \mathrm{Fe}_{12-2 x} \mathrm{O}_{19}(\mathrm{M}=\mathrm{Ba}, \mathrm{Sr})$ by floating zone and investigation of their magnetic and magnetoelectric properties Low Temperature Physics 43, 971 (2017); 10.1063/1.5001298

Effect of doping and annealing on crystal structure and magnetic properties of $\mathrm{La}_{1-x} \mathrm{Ag}_{\mathrm{X}} \mathrm{MnO}_{3}$ magnetic nanoparticles

Low Temperature Physics 43, 990 (2017); 10.1063/1.5001308

Magnetic ordering of anisotropic magnets due to the rotation of a magnetic field Low Temperature Physics 43, 960 (2017); 10.1063/1.5001296

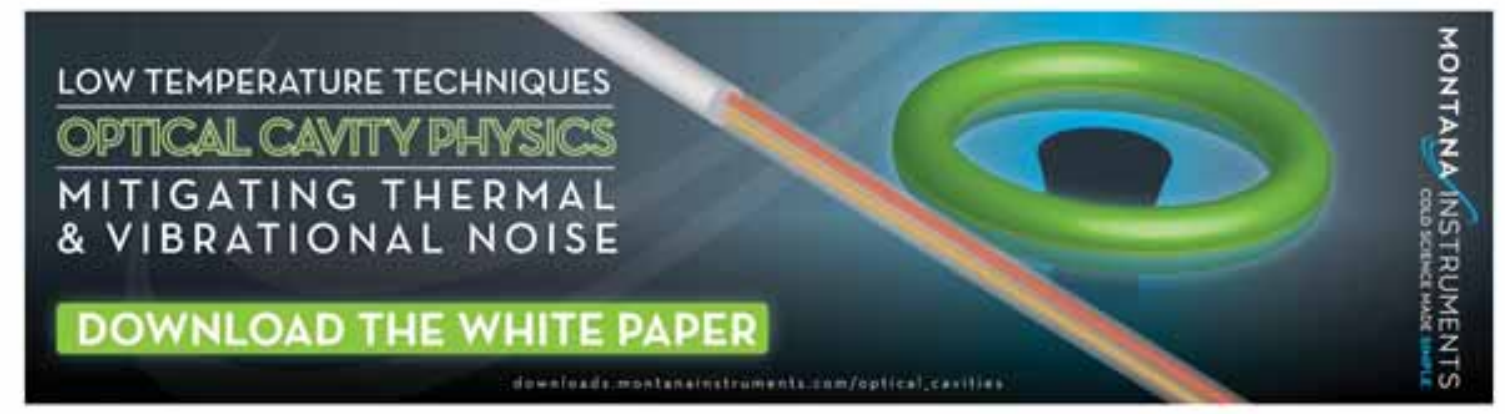




\title{
Classical and exotic magnetism: Recent advances and perspectives
}

\author{
Yuri Kivshar ${ }^{\mathrm{a})}$ \\ Nonlinear Physics Center, Research School of Physics and Engineering, Australian National University, \\ Canberra, ACT 2601, Australia
}

\section{Andrew P. Roberts}

Research School of Earth Sciences, Australian National University, Canberra, ACT 2601, Australia (Submitted February 6, 2017)

Fiz. Nizk. Temp. 43, 1119-1125 (August 2017)

\begin{abstract}
Motivated by the pioneering studies of Eremenko of magneto-optical effects in antiferromagnetic crystals, we describe an expansion of the horizons of classical magnetism to include study of Earth's magnetic field and natural environments. We also review briefly examples of exotic magnetism not directly associated with alignment of quantum spins in traditional ferromagnetic and antiferromagnetic crystalline structures. While many such cases have long been known, recent breakthroughs are associated with so-called optical magnetism that became a reality due to development of the field of metamaterials. Published by AIP Publishing. [http://dx.doi.org/10.1063/1.5001281]
\end{abstract}

\section{Introduction}

The phenomenon of magnetism is usually associated with specific states of crystalline solids in which electron spins within the material align in a specific direction. This classical approach was followed by V. Eremenko who more than 30 years ago undertook experimental studies of magneto-optical effects in antiferromagnetically ordered crystals. ${ }^{1}$

The extended zoo of magnetism also houses some interesting species in which classical magnetic systems have varying origins and applications. In recent years we have witnessed a renewed interest in the study of magnetic phenomena at different scales, ranging from the remarkable magnetar stars ${ }^{2}$ with the strongest magnetic fields in the Universe (typically $\left.10^{10}-10^{11} \mathrm{~T}\right)$, to nanoscale objects where the effective magnetic properties are induced by incoming laser radiation. Magnetic phenomena have become more complex or unusual, with spins arranged in complicated geometries or with magnetic responses created by optical fields through resonances that lead to unusual phenomena such as optical magnetism. In this brief review paper, we describe some examples of classical and exotic magnetic systems, mainly from our recent research activities, that we consider relevant to celebrating the pioneering research contributions of V. Eremenko into the physics of magnetic systems summarized by his colleagues and friends in this special issue.

First, we discuss aspects of classical magnetism associated with antiferromagnetic order, particularly in relation to deviations from perfect antiferromagnetism, in the fields of paleomagnetism $^{3}$ and environmental magnetism., ${ }^{4,5}$ These applications involve the study of rock and mineral magnetism to understand how magnetic rock-forming minerals record information about past variations in Earth's magnetic field or about changing natural environments.

Next, we address the recent and still somewhat controversial issue of so-called optical magnetism. It is well established that in the optical regime natural materials do not exhibit magnetic properties so that their magnetic permeability $\mu$ can be set to unity, i.e., $\mu=1$, as described in the famous textbook of Landau and Lifshitz. ${ }^{6}$ We argue that the use of metamaterials and engineered artificial metaatoms and metadevices $^{7}$ allows any value of $\mu$ to be produced to give a strong magnetic response by achieving resonances in structured systems made of nonmagnetic materials. ${ }^{8}$ Although magnetism in its conventional sense is not available at midIR and optical frequencies, it is possible to engineer the spatial dispersion and nonlocal electric effects so as to induce a strong magnetic dipole moment without other higher-order contributions, even though the materials involved do not have a microscopic magnetization and their magnetic permeability is strictly unitary. ${ }^{9}$

Finally, we demonstrate that some of the exotic magnetic structures produced by optically induced magnetic moments can possess antiferromagnetic order. This provides a direct all-optical analog to the crystalline antiferromagnetic compounds studied by V. Eremenko and his colleagues almost 30 years ago. ${ }^{1}$

\section{Paleomagnetism and environmental magnetism}

Magnetic signal recording in minerals. In paleomagnetism and environmental magnetism, we measure magnetic signals recorded by magnetically ordered rock-forming minerals to address research questions in geology, geophysics, and environmental science. In his Nobel Prize winning work on the magnetic structure of antiferromagnetic materials, Néel $^{10}$ postulated the existence of magnetic sublattices, which was later proven by neutron diffraction, where neighboring electron spins have a mutually antiparallel alignment so that their magnetic moments cancel completely (Fig. 1). True antiferromagnetism is common in rock-forming minerals, but the zero net spontaneous magnetization does not provide signals of interest in paleomagnetism and environmental magnetism. Furthermore, true ferromagnetism (Fig. 1) does not exist in rock-forming minerals at Earth's surface. The magnetizations of interest in paleomagnetism and environmental magnetism arise due to ferrimagnetism, which is a sub-category of antiferromagnetism in which the magnetic moments of the two magnetic sublattices are antiparallel but of unequal magnitude. Ferrimagnets behave macroscopically 
Ferromagnetism

\begin{tabular}{l}
\hline$\uparrow \uparrow \uparrow \uparrow \uparrow \uparrow \uparrow \uparrow \uparrow \uparrow \uparrow$ \\
$\uparrow \uparrow \uparrow \uparrow \uparrow \uparrow \uparrow \uparrow \uparrow \uparrow \uparrow \uparrow$ moment \\
\hline
\end{tabular}

Ferrimagnetism

\begin{tabular}{cccc}
\hline Cation & $\begin{array}{c}\text { Octahedral } \\
\text { (B sublattice) }\end{array}$ & $\begin{array}{c}\text { Tetrahedral } \\
\text { (A sublattice) }\end{array}$ & $\begin{array}{c}\text { Net magnetic } \\
\text { moment }\end{array}$ \\
\hline $\mathrm{Fe}^{3+}$ & $\uparrow \uparrow \uparrow \uparrow$ & $\downarrow \downarrow \downarrow \downarrow$ & $\begin{array}{c}\text { Complete } \\
\text { cancellation }\end{array}$ \\
$\mathrm{Fe}^{2+}$ & $\uparrow \uparrow \uparrow \uparrow \uparrow \uparrow \uparrow \uparrow \uparrow \uparrow$ & $-\uparrow \uparrow \uparrow$ \\
& $\uparrow \uparrow \uparrow \uparrow$ & - & $\uparrow \uparrow \uparrow$
\end{tabular}

Antiferromagnetism

\begin{tabular}{ccc}
\hline $\begin{array}{c}\text { Octahedral } \\
\text { (B sublattice) }\end{array}$ & $\begin{array}{c}\text { Tetrahedral } \\
\text { (A sublattice) }\end{array}$ & $\begin{array}{c}\text { Net magnetic } \\
\text { moment }\end{array}$ \\
\hline$\uparrow \uparrow \uparrow \uparrow$ & $\downarrow \downarrow \downarrow \downarrow$ & $\begin{array}{c}\text { Complete } \\
\text { cancellation }\end{array}$ \\
$\uparrow \uparrow \uparrow \uparrow$ & $\downarrow \downarrow \downarrow \downarrow$ & \\
\hline
\end{tabular}

Canted antiferromagnetism

\begin{tabular}{ccc}
\hline Octahedral & Tetrahedral & Net magnetic \\
(B sublattice) & (A sublattice) & moment \\
\hline
\end{tabular}
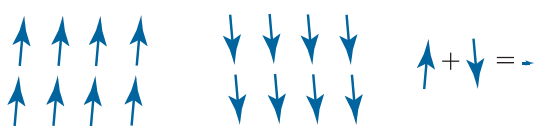

Fig. 1. Schematic two-dimensional representations of the main types of exchange-coupled magnetic spin structures. In a ferromagnetic structure, all magnetic moments are aligned, while in antiferromagnetic metal oxides nearest neighbor cations have antiparallel spin vectors and the spin structure consists of two equal and opposite magnetic sub-lattices with zero net moment. In ferrimagnetic metal oxides, as illustrated for the magnetic mineral magnetite, cations on non-equivalent sites are bonded with oxygen to produce a spin structure with two antiparallel but unequal magnetic sub-lattices that give rise to a net moment due to octahedrally coordinated $\mathrm{Fe}^{2+}$. Weak net magnetizations can also arise in imperfect antiferromagnetic structures due to lattice defects such as cation site vacancies (not illustrated), or due to canting of spins (e.g., in hematite). The degree of spin canting shown is $5^{\circ}$. Net magnetizations have been reported in materials with spin-canting of $<1^{\circ}$. The net magnetizations are small but measurable.

like ferromagnets, so they are often treated as equivalent, but the main difference is that ferrimagnets have a weaker net magnetization because of partial cancellation associated with the opposite but unequal sub-lattice magnetizations associated with the basic antiferromagnetic structure (Fig. 1). Ferrimagnetic order was invoked by $\mathrm{Ne}^{10}{ }^{10}$ to explain the magnetization of ferrites, the best-known example of which among magnetic minerals is magnetite $\left(\mathrm{Fe}_{3} \mathrm{O}_{4}\right)$ where the magnetic moments of an equivalent number of $\mathrm{Fe}^{3+}$ ions are aligned antiparallel so that the net magnetization arises from the magnetic moments of uncompensated $\mathrm{Fe}^{2+}$ ions (Fig. 1). Magnetizations of interest also arise due to other variations on the antiferromagnetic theme: where defects or vacancies in a crystal lattice give rise to incomplete cancellation of sublattice magnetic moments (defect antiferromagnetism) or where non-zero net magnetizations are achieved due to slight spin canting (canted antiferromagnetism; Fig. 1). The common magnetic iron oxide mineral hematite $\left(\alpha-\mathrm{Fe}_{2} \mathrm{O}_{3}\right)$ is a canted antiferromagnet. Magnetic signals due to deviations from classical antiferromagnetic order, thus, provide information that can be exploited to understand variations in Earth's magnetic field throughout most of our planet's history and to address wide-ranging environmental research questions as discussed briefly below.

Paleomagnetism: Earth's magnetic field is dominantly dipolar (Fig. 2), so that as early as $1600 \mathrm{AD}$, the personal physician of Queen Elizabeth I, William Gilbert, made the empirical deduction that Earth's magnetic field resembles that of a great magnet (he used lodestone (magnetite ore) in his experiments). This simple dipolar field geometry means that, when averaged over time, the inclination of the geomagnetic field is a simple function of latitude. When magnetic minerals record the orientation of the geomagnetic field at the time that their host rock formed, they provide a fossilized record of the ancient magnetic field. When rocks of varying ages are subjected to paleomagnetic analysis, the results do not provide a simple record of the inclination of the present day dipole field. Instead, for progressively older rocks from any continent, the pole calculated for a dipolar field will often lie far from geographic north. Calculated ancient pole positions are not random; they lie along coherent so-called apparent polar wander paths. The term apparent is used because the pole did not move; rather, it is the

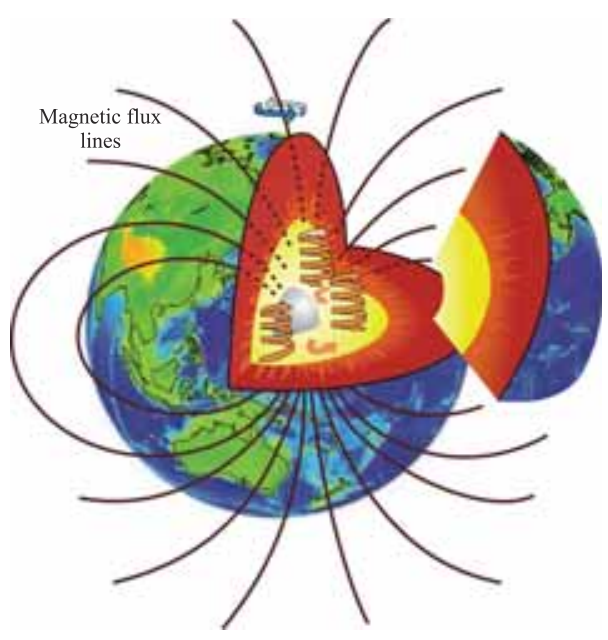

Fig. 2. Cut-away cartoon of Earth's interior with a view of the solid inner core (gray) and fluid outer core (yellow) from which geomagnetic flux lines emerge. The helical lines represent magnetic flux emerging from columnar convection cells that occur within the larger scale fluid flow in the outer core that drives the geodynamo. These cells transfer heat from the inner core-outer core boundary to the core-mantle boundary. The overall field is symmetrical about Earth's rotation axis. Over time, the field averages to a geocentric axial dipole, which is exploited in paleomagnetism to reconstruct the former positions of continental landmasses. 
continent that has moved with respect to the pole. The paleomagnetic record represented by the apparent polar wander path for a continent, thus, documents past movements of that continent. This information provided key evidence to confirm the theory of Continental Drift. It is not an overstatement to say that paleomagnetism has revolutionized our understanding of the Earth. It has been instrumental in establishing the plate tectonic paradigm, which provides a unifying theory for Earth science. Paleo-magnetism has also been used to demonstrate that the geomagnetic field has reversed polarity hundreds of times in Earth history (Fig. 3). Unlike the frequent and regular reversal of the sun's magnetic field (every 11 years), the process responsible for this flipping of geomagnetic polarity is stochastic. The randomly varying time intervals between successive reversals provides a bar-code-like pattern that has made it possible to develop a geomagnetic polarity timescale ${ }^{11}$ that is used to calibrate geological time by identifying magnetic polarity reversal patterns (Fig. 3) in geological sequences of lava flows or sedimentary rocks.

A fundamental aspect of paleomagnetism is that it provides an understanding of Earth's magnetic field and the processes that generate the field through a dynamo mechanism in which turbulent convection of an iron-nickel alloy in Earth's molten outer core produces a self-sustaining field (Fig. 2). Geomagnetic variations through geological time provide knowledge of a diverse range of field behaviors with variable time constants, including polarity reversals and excursions (thousands of years), and secular variations (years

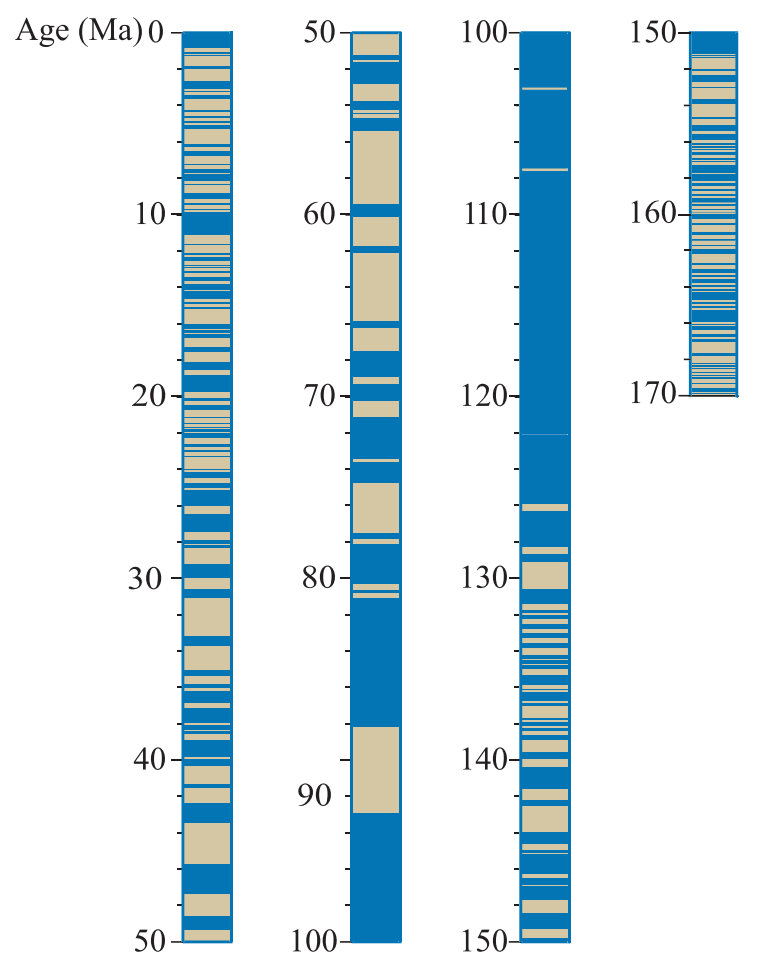

Fig. 3. Geomagnetic polarity timescale for the last $170 \times 10^{6}$ years (age is in million years ago, Ma). ${ }^{11}$ Blue $=$ normal polarity (i.e., like the present-day field); $\tan =$ reversed polarity (i.e., opposite to the present-day field). Reversal frequency has varied considerably over time; the field remained largely in a normal polarity configuration through the Cretaceous Normal Superchron $(\sim 126 \mathrm{Ma}$ to $\sim 93 \mathrm{Ma})$, but generally reverses polarity several times per million years. to millennia). The time interval between geomagnetic reversals generally ranges from hundreds of thousands of years to so-called "superchrons" when the field did not reverse polarity for tens of millions of years (Fig. 3). This indicates that phenomena with longer time constants also affect the field.

The geomagnetic field shields Earth from harmful cosmic radiation. Development of the early geomagnetic field is argued to have contributed to conditions that allowed evolution of life on, or at least contributed to the habitability of, our planet. ${ }^{12}$ All of this information about the Earth is the result of painstaking efforts by paleomagnetists to document information recorded by magnetic minerals. Magnetic fields are ubiquitous in the cosmos, yet field variations through time, and the valuable information they provide about cosmic evolution, are difficult to study because of the small and incomplete record provided by meteoritic materials. In contrast, Earth is a natural laboratory for analyzing the magnetic history of a planet through paleomagnetic analysis. Space limitations prevent us from elucidating the details of many fascinating phenomena discovered through paleomagnetic analysis of terrestrial and meteoritic materials. Readers who are interested in further details are referred to an excellent recent text that is available online. ${ }^{3}$

Environmental magnetism: In addition to their capacity to record information about ancient magnetic fields, magnetic minerals are responsive to changing environmental conditions. Environmental magnetism ${ }^{4,5}$ exploits the sensitivity of magnetic minerals to chemical reduction-oxidation processes at Earth's surface, which drive transformation of $\mathrm{Fe}^{2+}$ to $\mathrm{Fe}^{3+}$, and vice versa, in magnetic minerals that facilitates understanding of environmental processes. Wide-ranging processes are explored using environmental magnetism, which contributes to understanding the driving forces of environmental change. Instead of describing environmental magnetism in detail, we provide below a particularly clear example of its use in understanding climate variability.

Larrasoaña et al. ${ }^{13}$ used meteorological, satellite, and geochemical data to identify the eastern Sahara Desert north of the central Saharan watershed at $21^{\circ} \mathrm{N}$ as the source of windblown dust that accumulates in the Eastern Mediterranean Sea. In the hyper-arid Sahara, hematite forms though progressive dehydration and oxidation of other iron-bearing minerals. Regional aridity changes have driven variations in dust delivery from the northeastern Sahara to the Eastern Mediterranean Sea over millions of years in response to African monsoon variations, for which hematite is an excellent proxy. Hematite-based environmental magnetic records of Saharan dust deposition into the Eastern Mediterranean Sea, therefore, provide important insights into the long-term functioning of the African monsoon and the history of the Sahara Desert. ${ }^{13}$ Monsoons are controlled by variations in Earth's orbit that drive cyclical variations in the amount of incident solar radiation at Earth's surface. This magnetic record of dust inputs indicates that long-term African monsoon variations have driven cyclical climatic variations in North Africa, which undergoes large-scale changes from hyper-arid desert conditions to green Sahara episodes. ${ }^{13,14}$ Green Sahara episodes would have provided a much broader landscape that was suitable for hominin habitation and would have opened a migration pathway for our ancestors between Africa and Eurasia in contrast to modern hyper-arid conditions that provide a hostile environment for 
habitation and a major biogeographic barrier to migration. ${ }^{14}$ Orbital control of the monsoon is most clearly evident when the hematite dust record is subjected to band-pass filtering (Fig. 4). An inverse relationship exists between dust and orbital control because maximum dust production occurs during monsoon minima and vice versa. Age uncertainties for the sediment record mean that it is safest to undertake bandpass filtering over an age band for each orbital period. Dust variations occur in lock step with orbital variations (Fig. 4) and are statistically coherent with each other at the $99 \%$ significance level. ${ }^{13}$ While many examples of the use of magnetism for understanding environmental processes could be cited, such an immediate environmental response to Earth's orbital forcing of climate makes it clear that the magnetic properties of minerals can provide powerful information about environmental processes such as climate variability, monsoons, and desertification. The environmental magnetic record described here is due to canted antiferromagnetism in hematite, and gives an example of the diversity of applications that exploit antiferromagnetic structures.

\section{Optical magnetism}

Mie resonances and magnetic responses: We now move from classical to more exotic manifestations and applications of magnetism. Natural materials exhibit negligible magnetism at mid-IR and optical frequencies because the direct effects of optical magnetic fields on matter are much weaker than electric ones. However, in recent decades many researchers have sought to overcome this natural limitation by designing artificial subwavelength structures that allow a strong magnetic response, even if such structures are made of nonmagnetic materials. This progress has been possible due to advances in the field of metamaterials, which are composed of subwavelength elements that are often referred to as meta-atoms and the exotic states that they can support.

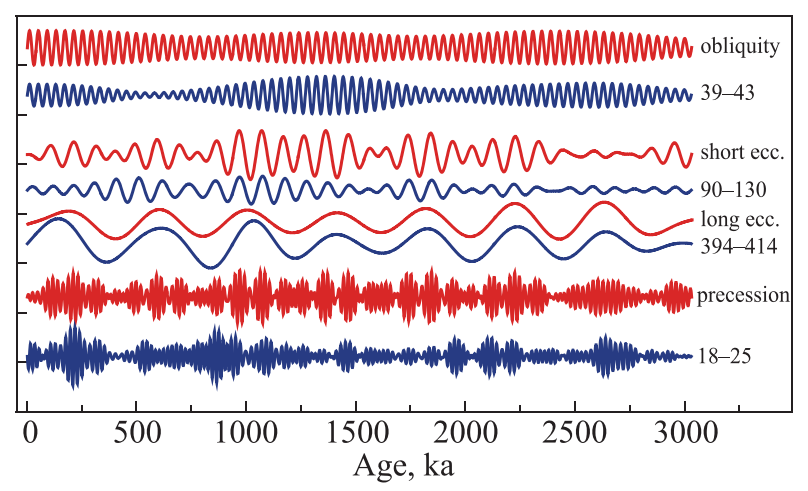

Fig. 4. Illustration of environmental magnetic analysis of windblown Saharan dust to understand climate forcing of dust deposition in marine sediments. The band-pass filtered dust record (blue; period bands are in thousands of years) of Larrasoaña et al. ${ }^{13}$ reflects an immediate response to insolation-driven African monsoon forcing at all major orbital periods (including precession, obliquity, and long and short eccentricity). The astronomical solution of Laskar et al. ${ }^{15}$ was band-pass filtered (red) using the same band as the dust record, and is normalized by the respective standard deviation. The band-pass-filtered dust signal is scaled identically for all period bands to conserve relative amplitude ratios between the signal components, and is multiplied by -1 because the insolation-driven monsoon response anticorrelates with dust production. Ages are in thousands of years before present $(\mathrm{ka})$.
One of the first examples of a meta-atom was the metallic split-ring resonator [Fig. 5(a)] where electrons oscillate in two parallel arms in the opposite direction to create an efficient magnetic response. Split-ring resonators were first introduced at microwave frequencies to realize artificial magnetic inclusions with subwavelength footprint, and were translated to optics by exploiting the plasmonic features of metallic nanoparticles. Such magnetic structures have now been realized in many nonmagnetic plasmonic structures ranging from nanobars ${ }^{16,17}$ and nanoparticle complexes, which are often called oligomers, ${ }^{18,19}$ to split-ring-based structures, ${ }^{20-22}$ and more complicated multilayered structures such as fishnet metamaterials with both elliptic and hyperbolic types of dispersion. $^{23}$

For many years, subwavelength localization of light in nanophotonics was associated with free electrons and electromagnetic waves at metallic interfaces. However, recent developments in the physics of high-refractive-index dielectric nanoparticles ${ }^{9}$ suggest an alternative mechanism of light localization via low-order dipole and multipole Mie resonances that may generate strong magnetic responses ${ }^{24}$ [Fig. 5(b)]. The physics of Mie resonances is usually associated with Rayleigh scattering and the colors of colloidal solutions with gold nanoparticles. However, a more recent understanding is that optical resonances of dielectric nanoparticles with high refractive index can also facilitate light manipulation below the free-space diffraction limit. ${ }^{9}$ To illustrate the properties of light scattering by nanoparticles, we consider a small nanodisk illuminated by a plane wave. Scattering by such a nanoparticle gives rise to strong resonances (Fig. 6).

Importantly, for dielectric particles we can observe both electric and magnetic responses of comparable strengths. A strong magnetic dipole resonance appears due to coupling of incoming light to the circular displacement currents of the electric field, owing to field penetration and phase retardation inside the particle. This occurs when the wavelength inside the particle becomes comparable to its spatial dimension such as radius, $2 R \approx \lambda / n$, where $n$ is the refractive index of the particle material, $R$ is the nanoparticle radius, and $\lambda$ is the wavelength of light. First, this type of geometric resonance suggests that a nanoparticle should have a relatively large refractive index in order to have resonances in visible and infrared spectral regions. Second, at the wavelength of a magnetic resonance, the excited magnetic dipole mode of a dielectric nanoparticle may become comparable or even stronger than the electric dipole response and make a major contribution to the scattering efficiency. Importantly, the

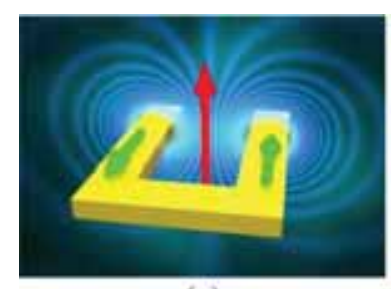

(a)

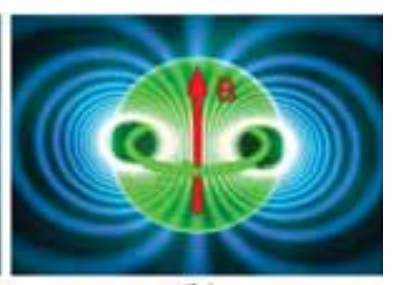

(b)
Fig. 5. Examples of optically induced magnetic dipole moments in metallic split-ring resonators (a) and dielectric nanoparticles with high refractive index (b). Green arrows indicate the direction of the electronic current and displacement current, respectively (courtesy of Miroshnichenko). 

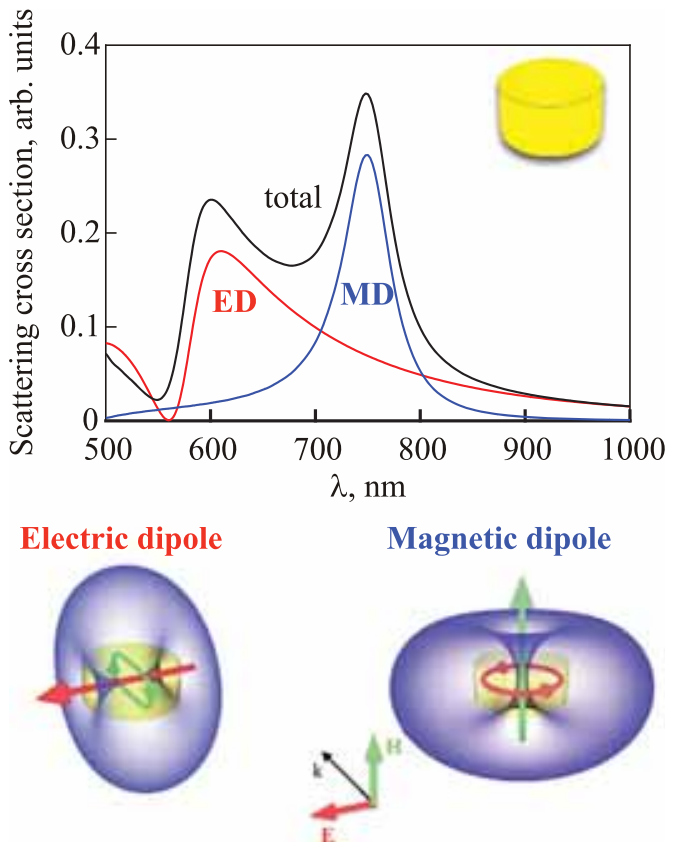

Fig. 6. Scattering efficiency for a silicon nanodisk with contributions from electric dipole (ED) and magnetic dipole (MD) Mie resonances, shown with the radiation patterns of two dipolar modes (Adopted from Ref. 8).

electric and magnetic dipoles are often perpendicular to each other (Fig. 6).

The term "displacement current" was considered for many years a mathematical curiosity introduced by Maxwell into his famous equations to provide continuity of current lines in nonconducting media, where the induction $D$ changes in time. Now, with resonant all-dielectric photonics driven by strong electric and magnetic dipole and multipole Mie resonances, the displacement current becomes a powerful tool of nanoscale photonics, and it helps to realize optical magnetism in low-loss, high-efficiency photonic structures. The spectral position of the magnetic dipole resonance of a spherical particle is approximately defined via a geometric resonance where the internal wavelength scales with the refractive index. Therefore, larger refractive indices are desirable from the point of view of metadevice dimensions and visible wavelength operation. The field enhancement and $Q$-factor of Mie resonances also benefit from large refractive indices of particles, with smaller radiation leakage. These considerations motivate the quest for materials with large refractive index.

In the visible and near-infrared spectral ranges, large permittivity is known to occur for semiconductors such as $\mathrm{Si}, \mathrm{Ge}$, AlGaAs, and others (Fig. 6). In the neighboring mid-infrared range, which is also of great interest in nanophotonics, narrowband semiconductors ( $\mathrm{Te}$ and $\mathrm{PbTe}$ ) and polar crystals such as $\mathrm{SiC}$ have been used in all-dielectric metadevices operating with Mie resonances. A pioneering milestone in optical magnetism was the experimental observation of a strong magnetic response in visible and infrared bands reported for spherical $\mathrm{Si}$ nanoparticles. ${ }^{25}$ This opened a way to study many interesting phenomena with high-refractive-index dielectric structures employed as building blocks for metadevices with inspiration from century-old studies of light scattering.

Optical antiferromagnetism: To reduce the interaction energy of atoms in magnetic solids, the spins of magnetic atoms can align in the opposite direction to that of their neighbors in some structures to give rise to the familiar antiferromagnetic order (Fig. 1). As discussed above, many compounds and oxides are antiferromagnetic, including $\mathrm{CoCO}_{3}$, $\mathrm{DyFe}_{3}$, and $\mathrm{Dy}_{3} \mathrm{Al}_{5} \mathrm{O}_{12}$, which were studied by Eremenko and Kharchenko. ${ }^{1}$ Antiferromagnetism is also associated with the expanding field of high-temperature superconductivity. ${ }^{26}$ Thus, the magnetic properties of antiferromagnetic structures remain of great scientific interest.

When meta-atoms that support optical magnetic dipoles are combined to create metamaterials or metasurfaces, the overall structure of the induced magnetic moments resembles ferromagnetic order (Fig. 1), as illustrated by two cases in Fig. 7. This is valid for both illustrated types of electromagnetic metasurfaces, where it is either induced in metallic meta-atoms by oscillation of free electrons or by a displacement current in dielectric meta-atoms with high refractive index. However, if we assemble hybrid structures with dissimilar elements with magnetic dipole moments of different origin (see Fig. 7), we expect that such hybrid meta-molecules with strong coupling of individual moments can create antiferromagnetic ordering of magnetic moments, as has been suggested recently from theoretical considerations. ${ }^{27}$

Experimental demonstration of antiferromagnetic ordering for a single meta-molecule and for metasurfaces has been reported recently, which establishes a new type of optically induced magnetism that resembles the staggered structure of spins in antiferromagnetically ordered materials. ${ }^{28}$ This demonstration was enabled by creating hybrid electromagnetic metasurfaces by assembling hybrid meta-atoms formed by metallic split-ring resonators and dielectric particles with high refractive index, both of which support optically induced magnetic dipole resonances of different origin. Each pair (or meta-molecule) is characterized by two interacting magnetic dipole moments with the distance-dependent magnetization resembling the spin exchange interaction in magnetic materials. By mapping directly the structure of the electromagnetic fields, it was demonstrated experimentally that strong coupling between optically induced magnetic moments of different origin can flip the magnetization orientation in a meta-molecule to create an antiferromagnetic

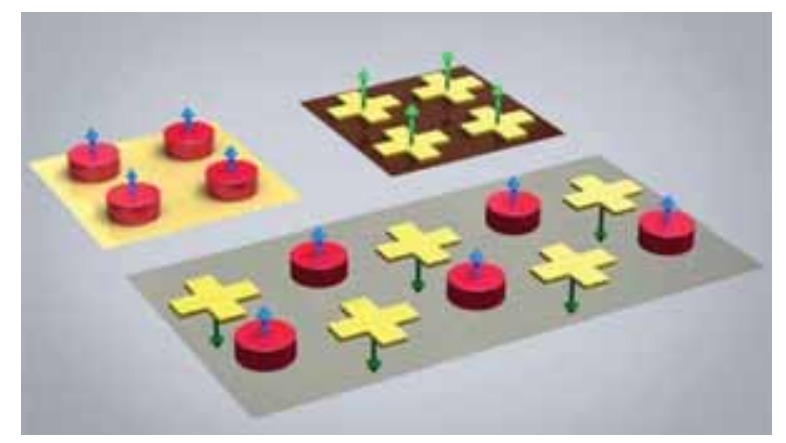

Fig. 7. Schematic illustration of magnetic dipole ordering in electromagnetic metasurfaces. A metasurface composed of identical elements (meta-atoms) resembles ferromagnetic ordering (Fig. 1) when magnetic dipole moments are induced (upper two cases). When combined, the two dissimilar types of meta-atoms with ferromagnetic ordering can create antiferromagnetic ordering (Fig. 1) with a staggered structure of optically induced magnetic dipole moments. The structure is one of several possible realizations of antiferromagnetic order (Adopted from Ref. 28). 
lattice of staggered magnetic moments in hybrid metasurfaces. $^{28}$ Such an approach can be further extended to achieve fine control of magnetic interaction with spectral tuning of multipolar coupling of different modes. Thus, hybrid metamaterials offer new opportunities to achieve unprecedented control of light-matter interactions.

\section{Conclusions and outlook}

Magnetism has changed our lives by giving us compasses that allow navigation. It lies behind many useful practical devices that help us to record sound and music, and allows us to store information. We believe that the importance of magnetism and magnetic phenomena will only grow, and will continue to bring new surprises in science and life.

Paleomagnetism has made paradigm-changing contributions to our understanding of Earth and its changing geography through time. Many interesting problems remain to be solved such as documenting the earliest geomagnetic fields and their potential relationship to early life on Earth. Likewise, paleomagnetic analysis of extraterrestrial materials is currently booming and is providing new insights into the workings of the early solar system and beyond. Environmental magnetic analysis is providing insights into wide-ranging environmental processes, which are important in understanding modern and ancient environmental change in response to natural and anthropogenic forcing.

On a different scene and scales, recent demonstrations of optical magnetism in many types of nanoscale structures are associated with new advances in nanotechnology, biotechnology, and the new field of meta-optics. More importantly, the study of optical magnetism with resonant highindex dielectric nanoparticles has emerged as a new direction in modern photonics. It originates from century-old studies of light scattering, and it brings various optically induced electric and magnetic dipole and multipole resonances into modern studies of metadevices driven by optical magnetic responses. Importantly, this also motivates the recently emerged field of multipolar nonlinear nanophotonics. ${ }^{29}$ These recent developments suggest intriguing opportunities for design of nonlinear subwavelength light sources with reconfigurable radiation characteristics and for engineering large effective optical nonlinearities at the nanoscale, which could have important implications for novel nonlinear photonic devices operating beyond the diffraction limit.

High-refractive-index nanophotonics is expected to complement or even substitute for different plasmonic components in a range of applications. All-dielectric resonant structures have many advantages, including resonant behavior and low energy dissipation into heat. Importantly, the existence of strong electric and magnetic dipole and multipole resonances can result in constructive or destructive interferences with unusual beam shaping, and may also lead to resonant enhancement of magnetic fields in dielectric nanoparticles that bring many novel functionalities for both linear and nonlinear regimes. We expect that many nanophotonic effects can be enhanced by magnetic resonances, including Raman scattering, magneto-optical responses, and nonlinear parametric interactions.

Y.K. acknowledges useful collaboration and discussions with Dr. Andrey Miroshnichenko and his help with the figures. This work was supported by the Australian Research Council.

a)Electronic addresses: Yuri.Kivshar@anu.edu.au and ysk@intemode.on.net

${ }^{1}$ V. V. Eremenko and N. F. Kharchenko, Phys. Rep. 155, 379 (1987).

${ }^{2}$ L. Kruesi, Phys. World 28, 36 (2015).

${ }^{3}$ L. Tauxe, Essentials of Paleomagnetism (University of California Press, 2010); see https://earthref.org/MagIC/books/Tauxe/Essentials/

${ }^{4}$ R. Thompson and F. Oldfield, Environmental Magnetism (Allen and Unwin, Winchester, MA, 1986).

${ }^{5}$ Q. S. Liu, A. P. Roberts, J. C. Larrasoaña, S. K. Banerjee, Y. Guyodo, L. Tauxe, and F. Oldfield, Rev. Geophys. 50, RG4002, doi:10.1029/ 2012RG000393 (2012).

${ }^{6}$ L. D. Landau and E. M. Lifshitz, Electrodynamics of Continuous Media (Pergamon Press, 1960).

${ }^{7}$ N. Zheludev and Y. S. Kivshar, Nat. Mater. 11, 16 (2012).

${ }^{8}$ Y. Kivshar and A. Miroshnichenko, Opt. Photonics News 28, 24 (2017).

${ }^{9}$ A. Kuznetsov, A. Miroshnichenko, M. Brongersma, Y. Kivshar, and B. Lukyanchuk, Science 354, aag2472 (2016).

${ }^{10}$ L. Néel, Ann. Phys. 12, 137 (1948).

${ }^{11}$ J. G. Ogg, "The geomagnetic polarity time scale," in The Geologic Time Scale, edited by F. M. Gradstein, J. G. Ogg, M. D. Schmitz, and G. M. Ogg (Elsevier, 2012), p. 85.

${ }^{12}$ J. A. Tarduno, E. G. Blackman, and E. E. Mamajek, Phys. Earth Planet. Inter. 233, 68 (2014).

${ }^{13}$ J. C. Larrasoana, A. P. Roberts, E. J. Rohling, M. Winklhofer, and R. Wehausen, Clim. Dyn. 21, 689 (2003).

${ }^{14}$ J. C. Larrasoaña, A. P. Roberts, and E. J. Rohling, PLoS One 8, e76514 (2013).

${ }^{15}$ J. Laskar, F. Joutel, and F. Boudin, Astron. Astrophys. 270, 522 (1993).

${ }^{16}$ W. Cai, U. K. Chettar, H.-K. Yuan, V. C. de Silva, A. V. Kildishev, V. P. Drachev, and V. M. Shalaev, Opt. Express 15, 3333 (2007).

${ }^{17}$ T. Pakizeh, A. Dmitriev, M. S. Abrishamian, N. Granpayeh, and M. Kall, J. Opt. Soc. Am. B 25, 659 (2008).

${ }^{18}$ A. Alu and N. Engheta, Phys. Rev. B 78, 085112 (2008).

${ }^{19}$ L. Sun, T. Ma, S.-C. Yang, D.-K. Kim, G. Lee, J. Shi, I. Martinez, G.-R. Yi, G. Shvets, and X. Li, Nano Lett. 16, 4322 (2016).

${ }^{20}$ Y. A. Urzhumov and G. Shvets, Solid State Commun. 146, 208 (2008).

${ }^{21}$ B. Kante, K. O'Brien, A. Niv, X. Yin, and X. Zhang, Phys. Rev. B 85, 041103 (2012).

${ }^{22}$ L. Qin, K. Zhang, R.-W. Peng, X. Xiong, W. Zhang, X.-R. Huang, and M. Wang, Phys. Rev. B 87, 125136 (2013).

${ }^{23}$ S. Kruk, Z. Wong, E. Pshenay-Severin, K. O’Brian, D. N. Neshev, Y. S. Kivshar, and X. Zhang, Nat. Commun. 7, 11329 (2016).

${ }^{24}$ R. Merlin, Proc. Nat. Acad. Sci. U. S. A. 106, 1693 (2009).

${ }^{25}$ A. I. Kuznetsov, A. E. Miroshnichenko, Y. H. Fu, J. Zhang, and B. Lukanchuk, Sci. Rep. 2, 492 (2012).

${ }^{26}$ I. Bozovic, X. He, J. Wu, and A. T. Bollinger, Nature 536, 309 (2016).

${ }^{27}$ A. E. Miroshnichenko, B. Lukyanchuk, S. A. Maier, and Y. S. Kivshar, ACS Nano 6, 837 (2012).

${ }^{28}$ A. E. Miroshnichenko, D. Filonov, B. Lukyanchuk, and Y. S. Kivshar, New J. Phys. (in press).

${ }^{29}$ D. Smirnova and Y. S. Kivshar, Optica 3, 1241 (2016).

This article was published in English in the original Russian journal. Reproduced here with stylistic changes by AIP Publishing. 